\title{
Thermal Issues in Casimir Forces Between Conductors and Semiconductors
}

\author{
K. A. Milton $*$ \\ H. L. Dodge Department of Physics and Astronomy, University of Oklahoma, Norman, OK 73019 \\ Iver Brevil团 and Simen Å. Ellingsent \\ Department of Energy and Process Engineering, Norwegian University of Science and Technology, N-7491 Trondheim, Norway
}

\begin{abstract}
The Casimir effect between metal surfaces has now been well-verified at the few-percent level experimentally. However, the temperature dependence has never been observed in the laboratory, since all experiments are conducted at room temperature. The temperature dependence for the related Casimir-Polder force between an atom and a bulk material has, in contrast, been observed between a BEC and a silica substrate, with the environment and the silica held at different temperatures. There is a controversy about the temperature dependence for the force between metals, having to do with the magnitude of the linear temperature term for both low and high temperature, the latter being most prominent at large distances. There are also related anomalies pertaining to semiconductors. The status of this controversy, and of the relevant experiments, are reviewed in this report.
\end{abstract}

PACS numbers: 11.10.Wx,05.70.Ce,42.50.Lc,78.20.Ce

\section{INTRODUCTION}

The Casimir effect, reflecting quantum vacuum fluctuations in the electromagnetic field in a region with material boundaries, has been studied both theoretically and experimentally since 1948 [1]. The forces between dielectric and metallic surfaces both plane and curved have been measured at the 10 to 1 percent level in a variety of room-temperature experiments, and remarkable agreement with the zero-temperature theory has been achieved. For reviews see [2 4].

In fitting the data various corrections due to surface roughness, patch potentials, curvature, and temperature have been incorporated. It is the temperature correction that is the subject of the present paper. Temperature dependence has been detected for the Casimir-Polder force between atoms in a Bose-Einstein condensate [5, 6], the theory of which was worked out in 7]. A recent experiment by the Yale group at large distance $(0.7-7 \mu \mathrm{m})$ [8] shows the theoretically expected reduction of the high$T$ effect for the Casimir force between metallic surfaces. See also [9].

Theoretically, there are subtle issues concerning thermodynamics and electrodynamics which have resulted in disparate predictions concerning the nature of these corrections. (An overview is given in [10].) However, a general consensus has emerged that the low-temperature correction to the Casimir effect is relatively large, and that the linear high-temperature effect should be reduced from the naive expectation by a factor of $1 / 2$, in accord with the recent Yale experiment [8]. (For a critique of this experiment, see [11].)

\footnotetext{
*Electronic address: milton@nhn.ou.edu

$\dagger$ Electronic address: iver.h.brevik@ntnu.no

${ }^{\ddagger}$ Electronic address: simen.a.ellingsen@ntnu.no
}

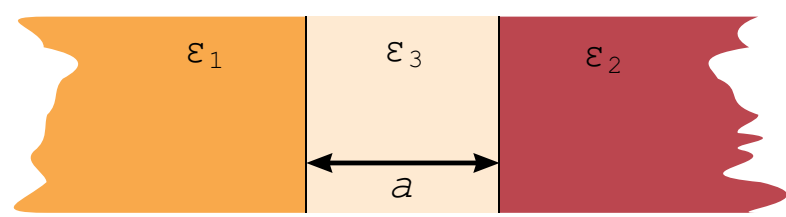

FIG. 1: Geometry of two semi-infinite parallel dielectric slabs, 1 and 2, separated by a third dielectric slab of thickness $a$. Each medium is characterized by a permittivity, $\varepsilon_{i}$, which is a function of the frequency.

\section{CONVENTIONAL APPROACH}

The zero-temperature Casimir effect between parallel conducting plates, or between parallel dielectrics, is very well understood, and is not controversial. The formula for the latter, which includes the former as a singular limit, may be derived by a multitude of formalisms [2[4, 12, 13]. For a system of parallel isotropic dielectric media, as shown in Fig. 1 characterized by a permittivity $(\varepsilon=\varepsilon(\omega))$

$$
\varepsilon(z)=\left\{\begin{array}{l}
\varepsilon_{1}, \quad z<0, \\
\varepsilon_{3}, \quad 0<z<a, \\
\varepsilon_{2}, \quad a<z,
\end{array}\right.
$$

the Lifshitz force per unit area on one of the surfaces is at zero temperature

$$
P^{T=0}=-\frac{1}{4 \pi^{2}} \int_{0}^{\infty} d \zeta \int_{0}^{\infty} d k_{\perp}^{2} \kappa_{3}\left(d^{-1}+d^{\prime-1}\right),
$$

where $\zeta$ is the imaginary frequency, $\zeta=-i \omega$, and the longitudinal wavenumber is

$$
\kappa_{i}=\sqrt{k_{\perp}^{2}+\zeta^{2} \varepsilon_{i}(i \zeta)},
$$

while the transverse electric (TE) and transverse magnetic (TM) Green's functions are characterized by the 
denominators

$$
\begin{aligned}
d & =\frac{\kappa_{3}+\kappa_{1}}{\kappa_{3}-\kappa_{1}} \frac{\kappa_{3}+\kappa_{2}}{\kappa_{3}-\kappa_{2}} e^{2 \kappa_{3} a}-1, \\
d^{\prime} & =\frac{\kappa_{3}^{\prime}+\kappa_{1}^{\prime}}{\kappa_{3}^{\prime}-\kappa_{1}^{\prime}} \frac{\kappa_{3}^{\prime}+\kappa_{2}^{\prime}}{\kappa_{3}^{\prime}-\kappa_{2}^{\prime}} e^{2 \kappa_{3} a}-1,
\end{aligned}
$$

respectively, where $\kappa_{i}^{\prime}=\kappa_{i} / \varepsilon_{i}$.

The attractive Casimir pressure between parallel perfectly conducting planes separated by a vacuum space of thickness $a$ is obtained by setting $\varepsilon_{1,2} \rightarrow \infty$ and $\epsilon_{3}=1$. In that case the TE and TM contributions are equal, and we have

$$
P_{C}=-\frac{1}{8 \pi^{2}} \int_{0}^{\infty} d \zeta \int_{\zeta^{2}}^{\infty} d \kappa^{2} \frac{4 \kappa}{e^{2 \kappa a}-1}=-\frac{\pi^{2} \hbar c}{240 a^{4}}
$$

which is Casimir's celebrated result [1].

The controversy surrounds the question of how to incorporate thermal corrections into the latter result. At first glance, the procedure to do this seems straightforward. It is well-known that thermal Green's functions must be periodic in imaginary time, with period $\beta=1 / T$ [14]. This implies a Fourier series decomposition, rather than a Fourier transform, where in place of the imaginary frequency integral above we have a sum over Matsubara frequencies

$$
\zeta_{m}^{2}=\frac{4 \pi^{2} m^{2}}{\beta^{2}}, \quad \int_{0}^{\infty} \frac{d \zeta}{2 \pi} \rightarrow \frac{1}{\beta} \sum_{m=0}^{\infty}
$$

the prime being an instruction to count the $m=0$ term in the sum with half weight. This prescription leads to the following formula for the Casimir pressure between perfect conductors at temperature $T,\left(t=\frac{4 \pi a}{\beta}\right)$

$$
P^{T}=-\frac{1}{4 \pi \beta a^{3}} \sum_{m=0}^{\infty} \int_{m t}^{\infty} y^{2} d y \frac{1}{e^{y}-1}
$$

From this it is straightforward to find the high and low temperature limits,

$$
\begin{aligned}
& P^{T} \sim-\frac{1}{4 \pi \beta a^{3}} \zeta(3)-\frac{e^{-t}}{2 \pi \beta a^{3}}\left(1+t+\frac{t^{2}}{2}\right), \beta \ll 4 \pi a, \\
& P^{T} \sim-\frac{\pi^{2}}{240 a^{4}}\left[1+\frac{16}{3} \frac{a^{4}}{\beta^{4}}-\frac{240}{\pi} \frac{a}{\beta} e^{-\pi \beta / a}\right], \beta \gg 4 \pi a .
\end{aligned}
$$

These are the results found by Lifshitz [15], Fierz [16], Sauer [17], and Mehra [18]. The two limits are connected by the duality symmetry found by Brown and Maclay [19]. The pressure may be obtained by differentiating the free energy,

$$
P=-\frac{\partial}{\partial a} F
$$

which takes the following form for low temperature (now omitting the exponentially small terms)

$$
F \sim-\frac{\pi^{2}}{720 a^{3}}-\frac{\zeta(3)}{2 \pi} T^{3}+\frac{\pi^{2}}{45} T^{4} a, \quad a T \ll 1,
$$

from which the entropy follows,

$$
S \sim-\frac{\partial}{\partial T} F \sim \frac{3 \zeta(3)}{2 \pi} T^{2}-\frac{4 \pi^{2}}{45} T^{3} a, \quad a T \ll 1,
$$

which vanishes as $T$ goes to zero, in accordance with the third law of thermodynamics, the Nernst heat theorem.

\section{EXCLUSION OF TE ZERO MODE}

However, there is something peculiar about the procedure adopted above for a perfect metal [20]. It has to do with the transverse electric mode of zero frequency, which we shall refer to as the TE zero mode. If we examine the zero frequency behavior of the reflection coefficients for a dielectric we see that providing $\zeta^{2} \varepsilon(i \zeta) \rightarrow 0$ as $\zeta \rightarrow 0$, the longitudinal wavenumber $\kappa_{i} \rightarrow k$ as $\zeta \rightarrow 0$, and hence $d \rightarrow \infty$ as $\zeta \rightarrow 0$. This means that there is no TE zero mode for a dielectric. This statement is not controversial. However, if a metal is modeled as the $\varepsilon \rightarrow \infty$ limit of a dielectric, the same conclusion would apply. Because that would spoil the concordance with the third law noted in the previous section, the prescription was promulgated [13, 15] that the $\varepsilon \rightarrow \infty$ limit be taken before the $\zeta \rightarrow 0$ limit. But, of course, a real metal is not described by such a mathematical limit, so we must examine the physics carefully.

A simple model for the dielectric function is the plasma dispersion relation,

$$
\varepsilon(\omega)=1-\frac{\omega_{p}^{2}}{\omega^{2}}
$$

where $\omega_{p}$ is the plasma frequency. For this dispersion relation, the condition $\zeta^{2} \varepsilon(i \zeta) \rightarrow 0$ fails to hold as $\zeta \rightarrow 0$, and the idealized prescription result, namely the contribution of the TE zero mode, follows.

However, real metals are not well described by this dispersion relation. Rather, there is no TE zero mode in the Drude model,

$$
\varepsilon(i \zeta)=1+\frac{\omega_{p}^{2}}{\zeta(\zeta+\gamma)}
$$

where the relaxation frequency $\gamma$ represents dissipation. The Drude model very accurately fits optical experimental data for the permittivity for $\zeta<2 \times 10^{15} \mathrm{rad} / \mathrm{s}$ [21, 22]. For gold, appropriate values of the parameters are $\omega_{p}=9.03 \mathrm{eV}, \gamma=0.0345 \mathrm{eV}$.

Let us review the argument by writing the Lifshitz formula at finite temperature in the form

$$
P^{T}=\sum_{m=0}^{\infty}{ }^{\prime} f_{m}=\int_{0}^{\infty} d m f(m)-\sum_{k=0}^{\infty} \frac{B_{2 k}}{(2 k) !} f^{(2 k-1)}(0),
$$


where the second equality uses the Euler-Maclaurin sum formula, in terms of

$$
f(m)=-\frac{1}{2 \pi \beta} \int_{0}^{\infty} d k_{\perp}^{2} \kappa\left(\zeta_{m}\right)\left(d_{m}^{-1}+d_{m}^{\prime-1}\right),
$$

where we assume that vacuum separates the two plates so $\kappa_{3}\left(\zeta_{m}\right)=\kappa\left(\zeta_{m}\right)=\sqrt{k_{\perp}^{2}+\zeta_{m}^{2}}$. Here the denominators are functions of $\zeta_{m}$. By changing the integration variable from $m$ to $\zeta_{m}$ we immediately see that the integral term in the Euler-Maclaurin sum formula corresponds precisely to the zero-temperature result.

One must, however, be careful in computing the low temperature corrections to this. One cannot directly expand the denominator $d$ in powers of $\zeta$ because the $k_{\perp}$ integral ranges down to zero. Let us rewrite the TE term there as follows:

$$
\begin{aligned}
& f^{(\mathrm{TE})}(m)=-\frac{1}{\pi \beta} \int_{2 m \pi / \beta}^{\infty} d \kappa \kappa^{2} \\
& \times\left\{\left[\frac{1+\sqrt{1+\zeta_{m}^{2}\left(\varepsilon\left(i \zeta_{m}\right)-1\right) / \kappa^{2}}}{1-\sqrt{1+\zeta_{m}^{2}\left(\varepsilon\left(i \zeta_{m}\right)-1\right) / \kappa^{2}}}\right]^{2} e^{2 \kappa a}-1\right\}^{-1}(3.5)
\end{aligned}
$$

Evidently, for the Drude model, or more generally, whenever

$$
\lim _{\zeta \rightarrow 0} \zeta^{2}[\varepsilon(i \zeta)-1]=0,
$$

the TE zero mode vanishes, $f^{(\mathrm{TE})}(0)=0$. However, it is important to appreciate the physical discontinuity between $m=0$ and $m=1$ for room temperature. At 300 $\mathrm{K}$, while $\zeta_{0}=0, \zeta_{1}=2 \pi T=0.16 \mathrm{eV}$, large compared the relaxation frequency $\gamma$. Therefore, for $m>0$,

$$
\begin{gathered}
-\frac{1}{\pi \beta} \int_{\zeta_{m}}^{\infty} d \kappa \kappa^{2}\left[\left(\frac{\sqrt{1+\omega_{p}^{2} / \kappa^{2}}+1}{\sqrt{1+\omega_{p}^{2} / \kappa^{2}}-1}\right)^{2} e^{2 \kappa a}-1\right]^{-1} \\
\approx-\frac{1}{\pi \beta} \int_{\zeta_{m}}^{\infty} d \kappa \kappa^{2} \frac{1}{e^{2 \kappa a}-1}
\end{gathered}
$$

provided the significant values of $\zeta_{m}$ and $\kappa$ are small compared to the plasma frequency $\omega_{p}$. This is just the ideal metal result. Insofar as this is accurate, this expression yields the low- and high-temperature corrections seen above. However, there is now a discontinuity in the function $f^{(\mathrm{TE})}$. As $\zeta_{m} \rightarrow 0$,

$$
f^{(\mathrm{TE})}(m) \rightarrow-\frac{1}{\pi \beta} \int_{0}^{\infty} d \kappa \frac{\kappa^{2}}{e^{2 \kappa a}-1}=-\frac{\zeta(3)}{4 \pi \beta a^{3}},
$$

rather than zero.

This implies an additional linear term in the pressure at low temperatures:

$$
P^{T} \sim P^{T=0}+\frac{\zeta(3)}{8 \pi a^{3}} T, \quad a T \ll 1 .
$$

Exclusion of the TE zero mode will also reduce the linear temperature dependence expected at high temperatures,

$$
P^{T} \sim-\frac{\zeta(3)}{8 \pi a^{3}} T, \quad a T \gg 1,
$$

one-half the usual ideal metal result seen in Eq. (2.8a).

\section{PROXIMITY FORCE APPROXIMATION}

Most experiments are carried out between a sphere (of radius $R$ ) and a plane. In this circumstance, if $R \gg a$, $a$ being the separation between the sphere and the plate at the closest point, the force may be obtained from the proximity force approximation (PFA) 23],

$$
\mathscr{F}=2 \pi R F(a),
$$

$F(a)$ being the free energy for the case of parallel plates separated by a distance $a$. Thus in the idealized description, the low temperature dependence including our linear term is for $a T \ll 1$,

$$
\mathscr{F} \sim-\frac{\pi^{3} R}{360 a^{3}}\left[1-\frac{45}{\pi^{3}} \zeta(3) a T+\frac{360}{\pi^{3}} \zeta(3)(a T)^{3}-16(a T)^{4}\right] .
$$

Since this conversion is trivial, in the following we will restrict attention to the straightforward parallel plate situation. (Recently, use of PFA has been superseded by numerical/analytical methods, e.g., multiple-scattering and boundary element techniques. See 24] for a review.)

These results are only approximate, because they assume the metal is ideal except for the exclusion of the TE zero. Elsewhere, we have referred to this model as the Modified Ideal Metal (MIM) model [25]. Evidently, for sufficiently low temperatures the approximation used here, that $\zeta_{1} \gg \gamma$, breaks down, the function $f(m)$ becomes continuous, and the linear term disappears. Indeed, numerical calculations based on real optical data for the permittivity show this transition. Because this linear behavior does not persist at arbitrarily small temperatures, it is clear that the conflict with the third law anticipated in the arguments in the previous section do not apply. In fact, as we shall see in Sec. VI the entropy does go to zero at zero temperature for any realistic metal. Only in special idealized model cases does the entropy problem persist, wherein $\zeta_{1} \gg \gamma$ remains true all the way to zero temperature; i.e., when $\gamma$ tends to zero as $\gamma \propto T$ or faster as $T \rightarrow 0$ [26, 27].

\section{KRAMERS-KRONIG RELATION}

As noted above, there are strong thermodynamic and electrodynamic arguments in favor of the exclusion of the TE zero mode. Essentially, the point is that a realistic physical system can have only one state of lowest energy. Electrodynamically, one can start from the 
Kramers-Kronig relation that relates the real and imaginary parts of the permittivity, required by causality, which can be written in the form of a dispersion relation for the electric susceptibility [28]

$$
\chi(\omega)=\frac{\omega_{p}^{2}}{4 \pi} \int_{0}^{\infty} d \omega^{\prime} \frac{p\left(\omega^{\prime}\right)}{\omega^{\prime 2}-(\omega+i \epsilon)^{2}} .
$$

If the spectral function $p\left(\omega^{\prime}\right) \geq 0$ is nonsingular at the origin, it is easily seen that $\omega^{2} \chi(\omega) \rightarrow 0$ as $\omega \rightarrow 0$, which as shown in Sec. III implies the absence of the TE zero mode. Conversely, $p\left(\omega^{\prime}\right)$ must have a $\delta$-function singularity at the origin to negate this conclusion. This would seem implausible for any but an overly idealized model. In contrast, in the Drude model the spectral function is non-singular, becoming singular only in the plasmamodel limit:

$$
p\left(\omega^{\prime}\right)=\frac{2}{\pi} \frac{\gamma}{\omega^{\prime 2}+\gamma^{2}} \rightarrow 2 \delta\left(\omega^{\prime}\right), \quad \gamma \rightarrow 0 .
$$

As noted above, use of the plasma model in the reflection coefficients would lead to the conventional temperature dependence, but this dispersion relation is inconsistent with real data. It may be argued [29] that in the ideal Bloch-Grüneisen model [30] the relaxation parameter goes to zero as $\gamma \propto T^{5}$ near zero temperature, thus causing the entropy to tend to a non-zero value at zero temperature. Physically this idealized case was shown to correspond to a glass-like state of frozen, randomly oriented bulk currents, whose ground state is strongly degenerate, hence retaining finite entropy in this limit without thereby violating the third law [31, 32].

More to the point, however, real materials exhibit scattering by impurities and certainly from boundaries, ${ }^{1}$ so that $\gamma$ remains finite. In any case, at sufficiently low temperatures the residual value of the relaxation parameter does not play a role, as the frequency characteristic of the anomalous skin effect becomes dominant [34]. Moreover, the Purdue group 35] also extrapolate the plasma formula from the infrared region down to zero frequency, whereas in fact frequencies very small compared to the frequency corresponding to the separation distance play a dominant role in the temperature dependence.

Finally, we emphasize that all present Casimir experiments are carried out at room temperature, where the known room temperature data are relevant.

\section{THIRD LAW OF THERMODYNAMICS}

The principal reason for the theoretical controversy has to do with the purported violation of the third law of

\footnotetext{
${ }^{1}$ Indeed, for very pure metals $\gamma$ is found experimentally to be sample-size dependent, and hence reaches a nonzero value even for an imperfection-free metal when its Bloch-Grüneisen electron mean free path becomes comparable to sample dimensions [33].
}

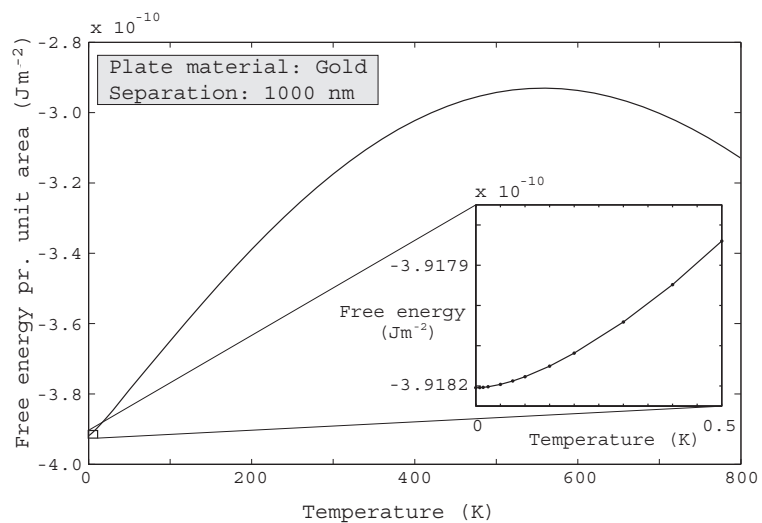

FIG. 2: Numerical evaluation of the free energy between two gold halfspaces as a function of temperature. The inset gives details for low $T$.

thermodynamics if the TE zero mode is not included. If ideal metal reflection coefficients are used otherwise (the MIM model) such a violation indeed occurs, because the free energy per unit area for small temperature then behaves like

$$
F=F_{0}+T \frac{\zeta(3)}{16 \pi a^{2}} .
$$

However, we and others have shown that for real metals, the free energy per area has a vanishing slope at the origin. Indeed, in the Drude model we have [4]

$$
F=F_{0}+T^{2} \frac{\omega_{p}^{2}}{48 \gamma}(2 \ln 2-1)
$$

for sufficiently low temperatures. The $T^{5 / 2}$ correction to this has been calculated in [36]. Figure 2, from that reference, shows a numerical evaluation of the free energy for gold, and how the low-temperature linear behavior gives way to a flat slope for very small temperatures. The exact mathematical requirements for the formal violation of the third law are reviewed in 37].

There is, however, an intermediate range of temperatures where it is expected that the entropy is negative. We do not believe that this presents a thermodynamic difficulty, and reflects the fact that the electrodynamic fluctuations being considered represent only part of the complete physical system [38]. It was also pointed out by Pitaevskii that the "Casimir entropy" is not a measure of the system's absolute entropy, but is to be understood as the difference between the (positive) entropies of an initial and final state, and there is no theorem dictating the sign of such a difference [39].

Independent arguments also lend support to our point of view. Jancovici and Šamaj [40] and Buenzli and Martin [41, 42] have examined the Casimir force between ideal-conductor walls with emphasis on the hightemperature limit. Not surprisingly, ideal inert boundary conditions are shown to be inadequate, and fluctuations 
within the walls, modeled by the classical Debye-Hückel theory, determine the high temperature behavior. The linear in temperature behavior of the Casimir force is found to be reduced by a factor of two from the behavior predicted by an ideal metal, just as seen above. This is precisely the signal of the omission of the $m=0 \mathrm{TE}$ mode. Thus, it is very hard to see how the corresponding modification of the low-temperature behavior can be avoided.

It is just the reduction of this dependence by the factor of $1 / 2$ that is seen in the Yale experiment [8].

\section{BOHR-VAN LEEUWEN THEOREM}

Bimonte [43] has suggested that the Bohr-van Leeuwen theorem, which says that classically at thermal equilibrium electromagnetic fields decouple from matter, explaining why normal metals do not exhibit strong diamagnetism, requires that the TE reflection coefficient vanishes as zero frequency. Plasma-like prescriptions are thus ruled out. The Bohr-van Leeuwen theorem does not apply to magnetic materials or superconductors, since quantum effects dominate there. Thus experiments on these materials might reveal quite different behaviors. Nevertheless, the Bohr-van Leeuwen theorem would seem a more natural criterion to turn to than the third law, for systems where magnetic effects are negligible at room temperature; the former applies in the high temperature limit which is exactly where the two Casimir predictions differ the most, whereas the latter applies only as the temperature tends to zero, a situation very far from laboratory conditions in any experiment reported to date.

\section{EXPERIMENTAL CONSTRAINTS}

We have marshalled theoretical arguments that seem to us quite overwhelming in favor of the absence of the TE zero mode in the temperature dependence of the Casimir force between real metal plates, which seem to imply unambiguously that there should be large $(\sim 15 \%)$ thermal corrections to the Casimir force at separations of order 1 micrometer. Calculations based on this theory, and using real optical data for aluminum, for example, have been given, as shown in Fig. 3 for aluminum plates, taken from [10]. The difficulty is that, experimentally, it is not easy to perform Casimir force measurements at other than room temperature, so current constraints on the theory all come from room temperature experiments. Then all one can do is compare the theory at room temperature with the experimental results, which must be corrected for a variety of effects, such as surface roughness, finite conductivity, and patch potentials. A deviation between the zero temperature theory and room temperature observations is a measure of the temperature correction.

Figure 4 shows the expected behavior of the pressure as a function of plate separation $a$ at room temperature.

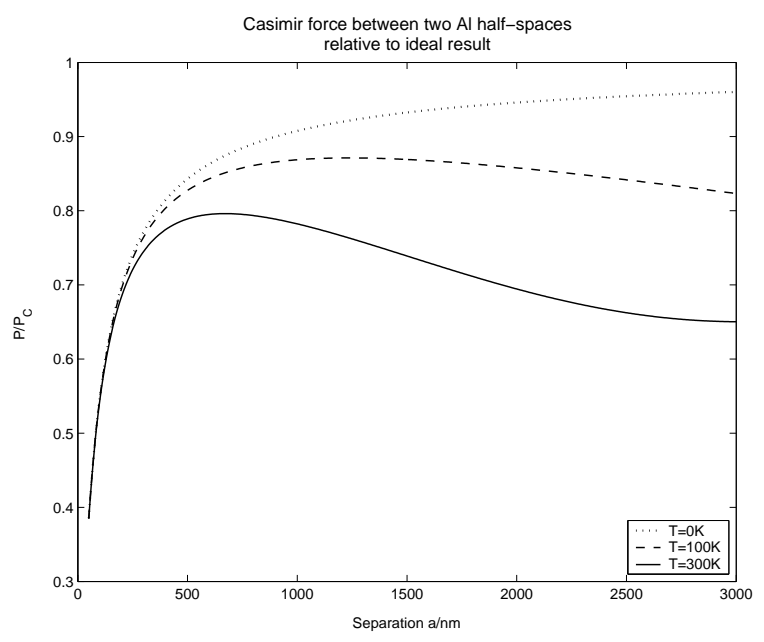

FIG. 3: Temperature dependence of the Casimir force between aluminum plates. The abscissa is the separation in nanometers.

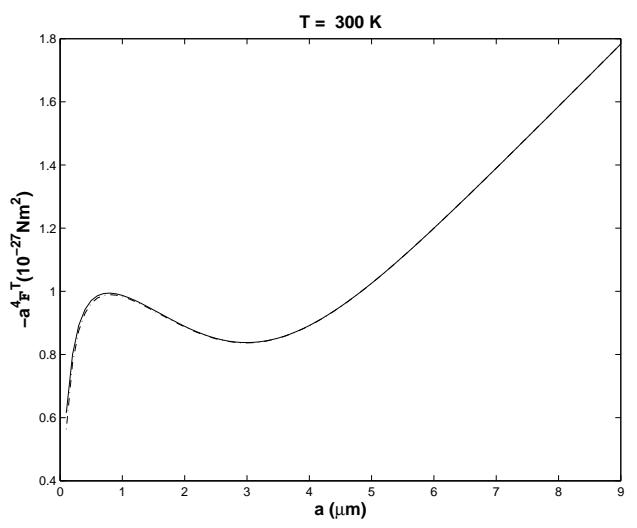

FIG. 4: The pressure between two gold plates multiplied $a^{4}$, plotted versus the plate separation $a$ for room temperature. Data for the permittivity is taken from [21, 22]. This figure is taken from [25].

Since, approximately, the dependence is through the dimensionless factor (in natural units) $a T$, the high temperature linear behavior is translated into a linear behavior in the separation. (The short distance linear regime is also seen with the expected slope.)

The temperature correction is evidently relatively largest at the largest separations, where, unfortunately, the total Casimir force is weakest. Lamoreaux's new experiment [8] is conducted at the $0.7-7 \mu \mathrm{m}$ scale, which is where the linear high-temperature result is dominant. At these large distances, the Casimir force is very small, and is overwhelmed by patch potentials. Nevertheless, after the patch-potential effect is extracted and subtracted from the data, the effect is to strongly favor the Drudemodel, that is, exclusion of the TE zero mode, as illustrated in Fig. [4. The procedures and conclusions of [8] are strongly critized in 11.

It is the most precise experiments of the Purdue group 
[44, 45] that claim the extraordinarily high precision to be able to see our effect at distances as small as 160 $\mathrm{nm}$. Indeed, they see no deviation from the corrected zero-temperature Lifshitz theory using optical permittivities, and hence they assert that our theory (the "Drude model") is decisively ruled out. The effect we predict for the temperature correction is only at the $1 \%$ level at a distance of $160 \mathrm{~nm}$, so the measurement must be performed to that accuracy to see the effect there. (For the usually employed sphere-plate configuration, $\Delta \mathscr{F} / \mathscr{F} \approx 2.5 \%$ at $a=160 \mathrm{~nm}$.) Taking the reported accuracy at face value, the nondissipative plasma-model approach is favored at a very high confidence level. While doubt has been aired on a general basis that a $1 \%$ accuracy can be possible [46], we are not aware of any criticism regarding concrete points in Decca's impressive experiment, and the results found must be regarded as something of a mystery.

Recently, there has also been a report from the Riverside group [47], claiming that the Drude model is also disfavored, although not at as high a level of significance. We cannot comment further on this result at present, except to note that the theoretical support for both the Purdue and the Riverside experiments is identical.

\section{ANOMALY FOR SEMICONDUCTORS}

Mostepanenko and Klimchitskaya have also claimed there is a similar anomaly, now affecting the TM reflection coefficient, for dielectrics and semiconductors 48]. Here is a simple way to understand that argument [49]. Suppose we model a dielectric with some small conductivity by the permittivity function

$$
\varepsilon(i \zeta)=1+\frac{\varepsilon_{0}-1}{1+\zeta^{2} / \omega_{0}^{2}}+\frac{4 \pi \sigma}{\zeta} .
$$

(The only essential point is that as $\zeta \rightarrow 0, \varepsilon \rightarrow \varepsilon_{0}$ if $\sigma=$ 0 , otherwise $\varepsilon \rightarrow \infty$.) The Casimir (Lifshitz) pressure is given by

$$
P=-\frac{T}{\pi} \sum_{i=\mathrm{TE}, \mathrm{TM}} \sum_{m=0}^{\infty}, \int_{\zeta_{m}}^{\infty} d \kappa \kappa^{2} \frac{1}{\left(r^{i}\right)^{-2} e^{2 \kappa a}-1},
$$

where the reflection coefficients are

$$
\begin{aligned}
r^{\mathrm{TE}} & =\frac{-\sqrt{1+\frac{\zeta^{2}}{\kappa^{2}}(\varepsilon(i \zeta)-1)}+1}{\sqrt{1+\frac{\zeta^{2}}{\kappa^{2}}(\varepsilon(i \zeta)-1)}+1}, \\
r^{\mathrm{TM}} & =\frac{-\sqrt{1+\frac{\zeta^{2}}{\kappa^{2}}(\varepsilon(i \zeta)-1)}+\varepsilon(i \zeta)}{\sqrt{1+\frac{\zeta^{2}}{\kappa^{2}}(\varepsilon(i \zeta)-1)}+\varepsilon(i \zeta)} .
\end{aligned}
$$

For the case of an ideal metal, it was $r^{\mathrm{TE}}$ which was discontinuous:

$$
r^{\mathrm{TE}}(\zeta=0)=0, \quad \lim _{\zeta \rightarrow 0} r^{\mathrm{TE}}=-1,
$$

so this gave a linear temperature term when the sum over Matsubara frequencies is converted to an integral according to the Euler-Maclaurin formula, for example. For a dielectric the TE reflection coefficient is continuous, but if there is vanishingly small (but not zero) conductivity the TM coefficient is discontinuous:

$$
r^{\mathrm{TM}}(\zeta=0)=1, \quad \lim _{\zeta \rightarrow 0} r^{\mathrm{TM}}=\frac{\varepsilon_{0}-1}{\varepsilon_{0}+1} .
$$

This leads to a linear $T$ term in the pressure:

$$
\begin{aligned}
P_{T}^{\mathrm{TM}} & =\frac{T}{2 \pi} \int_{0}^{\infty} d \kappa \kappa^{2}\left[\frac{1}{\left(\frac{\varepsilon_{0}+1}{\varepsilon_{0}-1}\right)^{2} e^{2 \kappa a}-1}-\frac{1}{e^{2 \kappa a}-1}\right] \\
& =\frac{T}{2 \pi} \sum_{n=1}^{\infty}\left[\left(\frac{\varepsilon_{0}-1}{\varepsilon_{0}+1}\right)^{2 n}-1\right] \int_{0}^{\infty} d \kappa \kappa^{2} e^{-2 n \kappa a} \\
& =\frac{T}{8 \pi a^{3}}\left[\operatorname{Li}_{3}\left(\left[\frac{\varepsilon_{0}-1}{\varepsilon_{0}+1}\right]^{2}\right)-\zeta(3)\right]
\end{aligned}
$$

where the logarithmic integral function is

$$
\operatorname{Li}_{n}(\xi)=\sum_{k=1}^{\infty} \frac{\xi^{k}}{k^{n}}
$$

Note that the linear term vanishes for $\varepsilon_{0} \rightarrow \infty$. The free energy term $F$ is obtained from this by multiplying by $a / 2$. Thus at zero temperature, the entropy is nonzero,

$$
S=-\left(\frac{\partial F}{\partial T}\right)_{V}=-\frac{1}{16 \pi a^{2}}\left[\operatorname{Li}_{3}\left(\left[\frac{\varepsilon_{0}-1}{\varepsilon_{0}+1}\right]^{2}\right)-\zeta(3)\right],
$$

as found in [48], which again appears to violate the third law of thermodynamics, stating that the entropy of a nondegenerate system must vanish at zero temperature.

We believe that real materials cannot undergo this violation, and that, properly treated, the entropy must vanish at zero temperature. The details of this assertion, however, have not been worked out to our knowledge. In materials with a very small concentration of free charges, it seems highly unlikely that a simple, local, Drude-type model can provide an adequate description of reflectivity [26], and salvation may well be found to lie in a complete inclusion of spatial dispersion effects.

Three simultaneous and very similar efforts were made in this direction [50 52]. These partly seek to bridge the gap between the predictions of a naive Drude-like model (9.1) and an experiment performed by Mohideen's group [53, 54]. In that experiment, the carrier concentration of a semiconductor was increased by laser illumination, and the observed behaviour of the Casimir force is surprising at first sight: 1) When the carrier concentration is high, the conductivity must be included if the simple model (9.1) is used. 2) When the carrier density is low (but nonzero), the conductivity must be excluded in order to reproduce measured data. In light of the spatial dispersion theory based on Debye-Hückel screening 
50 52, 55], such behavior is not so surprising, however, although these theories fail to reproduce the data perfectly. Unlike the mystery of Decca's experiment with metal surfaces, the experimental result here appears intuitively reasonable, but a complete theoretical description without any ad hoc prescriptions is still lacking.

\section{REAL TEMPERATURE EXPERIMENTS}

In any case, it would seem imperative to perform experiments at different temperatures in order to provide evidence for or against temperature dependence of Casimir forces. However, such experiments are extremely difficult, and, for example, Onofrio has abandoned his attempt to do such a measurement (private communication). For example, the increase of temperature from 300 $\mathrm{K}$ to $350 \mathrm{~K}$ should reduce the force by about $2.5 \%$, for $\mathrm{Au}-\mathrm{Au}$ plates when the separation is 1.0 micrometer.

Even though the new Yale experiment [8] is consistent with the revised theory, it needs to be independently con- firmed, especially in view of the very large electrostatic corrections that must be supplied. Also, direct measurement of the temperature dependence would be highly beneficial. (The only direct temperature measurement of temperature dependence is that for the $\mathrm{CP}$ force on a BEC of Rb atoms [6].) We encourage experimentalists to redouble their efforts, for the issues involved touch at the heart of our fundamental theoretical understanding of electrodynamics, statistical mechanics, and quantum field theory.

\section{Acknowledgments}

The work of KAM was supported by grants from the US National Science Foundation and the US Department of Energy. We thank the organizers of the FQMT11 conference in Prague, Vaclav Spicka, Theo Nieuwenhuizen, and Peter Keefe, for putting together such a successful and interdisciplinary meeting.
[1] H. B. G, Casimir, Proc. K. Ned. Akad. Wet., 51, 793 (1948).

[2] M. Bordag, G. L. Klimchitskaya, U. Mohideen and V. M. Mostepanenko, Advances in the Casimir effect, (Oxford University Press, International series of monographs on physics. 145, 2009)

[3] K. A. Milton, The Casimir effect: Physical manifestations of zero-point energy (World Scientific, River Edge, NJ, 2001).

[4] K. A. Milton, J. Phys. A 37, R209 (2004).

[5] D. M. Harber, J. M. Obrecht, J. M. McGuirk and E. A. Cornell, Phys. Rev. A 72, 033610 (2005).

[6] J.M. Obrecht, R.J. Wild, M. Antezza, L.P. Pitaevskii, S. Stringari, and E.A. Cornell, Phys. Rev. Lett. 98, 063201 (2007).

[7] M. Antezza, L. P. Pitaevskii, S. Stringari and V. B. Svetovoy, Phys. Rev. A 77, 022901 (2008).

[8] A. O. Sushkov, W. J. Kim, D. A. R. Dalvit, and S. K. Lamoreaux Nature Physics 7, 230 (2011).

[9] K. A. Milton, Nature Physics 7, 190 (2011).

[10] I. Brevik, S. A. Ellingsen, and K. A. Milton, New J. Phys. 8, 236 (2006).

[11] G. L. Klimchitskaya, M. Bordag, and V. M. Mostepanenko, Int. J. Mod. Phys. A 27, 1260012 (2012).

[12] I. D. Dzyaloshinskii, E. M. Lifshitz, and L. P. Pitaevskii, Usp. Fiz. Nauk 73, 381 (1961) [Eng. Trans.: Sov. Phys. Usp. 4, 153 (1961)].

[13] J. Schwinger, L. L. DeRaad, Jr., and K. A. Milton, Ann. Phys. (N.Y.) 115, 1 (1978).

[14] P. C. Martin and J. Schwinger, Phys. Rev. 115, 1342 (1959).

[15] E. M. Lifshitz, Zh. Eksp. Teor. Fiz 29, 94 (1956) [Eng. Trans.: Sov. Phys. JETP 2, 73 (1956)].

[16] M. Fierz, Helv. Phys. Acta 33, 855 (1960).

[17] F. Sauer, Ph.D. Dissertation, Göttingen, 1962.

[18] J. Mehra, Physica 37, 145 (1967).
[19] L. S. Brown and G. J. Maclay, Phys. Rev. 184, 1272 (1969).

[20] M. Boström and Bo E. Sernelius, Phys. Rev. Lett. 84, 4757 (2000).

[21] A. Lambrecht and S. Reynaud, Eur. Phys. J. D 8, 309 (2000).

[22] A. Lambrecht and S. Reynaud, Phys. Rev. Lett. 84, 5672 (2000).

[23] B. V. Derjaguin, Kolloid Z. 69, 155 (1934).

[24] A. Lambrecht, A. Canaguier-Durand, R. Guérout, and S. Reynaud, Chap. 4, p. 97; S. J. Rahi, T. Emig, and R. L. Jaffe, Chap. 5, p. 129; S. G. Johnson, Chap. 6, p. 175, in Casimir Physics, Lecture Notes in Physics, vol. 834, ed. D. Dalvit, P. Milonni, D. Roberts, and F. da Rosa, (Springer, Heidelberg, 2011).

[25] J. S. Høye, I. Brevik, J. B. Aarseth, and K. A. Milton, Phys. Rev. E 67, 056116 (2003).

[26] S. Å. Ellingsen, Phys. Rev. E 78, 021120 (2008).

[27] F. Intravaia and C. Henkel, J. Phys. A 41, 164018 (2008).

[28] J. Schwinger, L. L. DeRaad, Jr., K. A. Milton, and W.y. Tsai, Classical Electrodynamics (Perseus Books, New York, 1998), Eq. (51.27).

[29] V. B. Bezerra, G. L. Klimchitskaya, V. M. Mostepanenko, and C. Romero, Phys. Rev. A 69, 022119 (2004).

[30] Handbook of Physics, edited by E.U. Condon and H. Odishaw, 2nd ed. (McGraw-Hill, New York, 1967), Eq. (6.12).

[31] F. Intravaia and C. Henkel, Phys. Rev. Lett. 103, 130405 (2009).

[32] F. Intravaia, S. Å. Ellingsen, and C. Henkel, Phys. Rev. A 82, 032504 (2010).

[33] G. Burns, Solid State Physics, Int. ed. (Academic Press, London, 1985), Chap. 9.

[34] V. B. Svetovoy and R. Esquivel, Phys. Rev. E 72, 036113 (2005).

[35] V. B. Bezerra, R. S. Decca, E. Fischbach, B. Geyer, G. L. 
Klimchitskaya, D. E. Krause, D. López, V. M. Mostepanenko, and C. Romero, Phys. Rev. E 73, 028101 (2006).

[36] J. S. Høye, I. Brevik, S. A. Ellingsen and J. B. Aarseth, Phys. Rev. E 75, 051127 (2007).

[37] S. A. Ellingsen, I. Brevik, J. S. Høye, and K. A. Milton, J. Phys.: Conf. Series 161, 012010 (2009).

[38] I. Brevik, J. B. Aarseth, J. S. Høye, and K. A. Milton, Phys. Rev. E 71, 056101 (2005).

[39] L. P. Pitaevskii, in Proceedings of QFEXT09, ed. K. A. Milton and M. Bordag (World Scientific, Singapore, 2010), p. 227 arXiv:1010.0537.

[40] B. Jancovici and L. Šamaj, Europhys. Lett. 72, 35 (2005).

[41] P. R. Buenzli and Ph. A. Martin, Europhys. Lett. 72, 42 (2005).

[42] P. R. Buenzli and Ph. A. Martin, Phys. Rev. E 77, 011114 (2008).

[43] G. Bimonte, Phys. Rev. A 79, 042107 (2009).

[44] R.S. Decca, D. López, E. Fischbach, G.L. Klimchitskaya, D.E. Krause, and V.M. Mostepanenko, Phys. Rev. D 75, 077101 (2007).

[45] R.S. Decca, D. López, E. Fischbach, G.L. Klimchitskaya, D.E. Krause, and V.M. Mostepanenko, Eur. Phys. J. C
51, 963 (2007).

[46] S.K. Lamoreaux, Chap. 7, p. 219, in Casimir Physics, Lecture Notes in Physics, vol. 834, ed. D. Dalvit, P. Milonni, D. Roberts, and F. da Rosa (Springer, Heidelberg, 2011).

[47] C.-C. Chang, A. A. Banishev, R. Castillo-Garza, G. L. Klimchitskaya, V. M. Mostepanenko, and U. Mohideen, Phys. Rev. B 85, 165443 (2012).

[48] B. Geyer, G. L. Klimchitskaya, and V. M. Mostepanenko, Phys. Rev. D 72, 085009 (2005).

[49] S. A. Ellingsen, I. Brevik, J. S. Høye, and K. A. Milton, Phys. Rev. E 78, 021117 (2008).

[50] L. P. Pitaevskii, Phys. Rev. Lett. 101, 163202 (2008).

[51] D. A. R. Dalvit and S. K. Lamoreaux, Phys. Rev. Lett. 101, 163202 (2008).

[52] V. B. Svetovoy, Phys. Rev. Lett. 101, 163603 (2008).

[53] F. Chen, G.L. Klimchitskaya, V.M. Mostepanenko, and U. Mohideen, Optics Express 15, 4823 (2007).

[54] F. Chen, G. L. Klimchitskaya, V. M. Mostepanenko, and U. Mohideen, Phys. Rev. B 76, 035338 (2007).

[55] J. S. Høye and I. Brevik, Phys. Rev. E 80, 011104 (2009). 